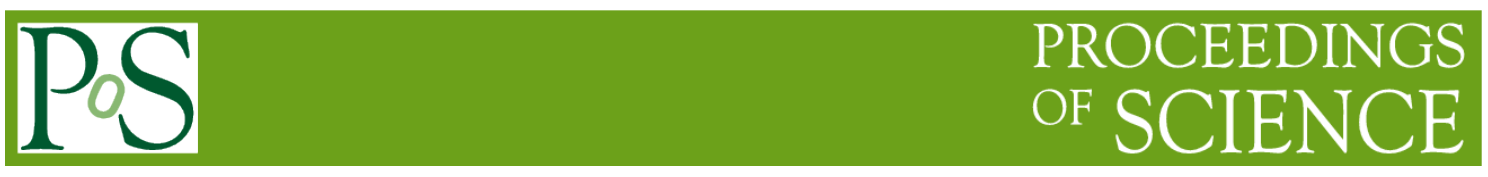

\title{
Optimization of the Liquid Scintillator Composition
}

\author{
A. Batyrkhanov ${ }^{1}$, D. Beznosko, A. lakovlev, K. Yelshibekov \\ Nazarbayev University \\ 53 Kabanbai Batyr ave, Astana, KZ \\ E-mail: ayan. batyrkhanov@nu.edu.kz
}

\begin{abstract}
Nowadays, many particle detectors use liquid scintillator (LS) as a detection medium. In particular, Water-based Liquid Scintillator (WbLS) that is a new material currently under development. It is based on the idea of dissolving the organic scintillator in water using special surfactants. This material strives to achieve the novel detection techniques by combining the Cherenkov and scintillation light, as well as the total cost reduction compared to pure liquid scintillator.
\end{abstract}

An important part of either the pure LS or WbLS production is to choose the right fluor and shifter and their concentrations. The choice affects the spectral distribution of the light output and the detection efficiency as each photodetector has its own spectral sensitivity region. This work presents the results of the study on the pseudocumen (PC) based LS with the PPO and POPOP/MSB as a fluor and shifters of choice. Both the total light yield and the spectral differences in the outputs with different amounts of components are shown. This study can be applied to plastic scintillators as well.

38th International Conference on High Energy Physics

3-10 August 2016

Chicago, USA

\section{${ }^{1}$ Speaker}




\section{Introduction}

Liquid scintillator is a charged particle detection medium that consists of an aromatic organic solvent base in which additional scintillator components are dissolved. It has the advantages over inorganic scintillators such as lower cost and fast light yield that is similar to plastic scintillators. Both of these types of organic scintillator and their detection properties are described in [1]. Liquid scintillator normally includes two additional components (dopants): fluor and shifter. They adjust the output wavelength for readout by a typical Photomultiplier Tube (PMT) or for usage with the wavelength shifting fibers. The process of optimization is done to minimize the amount of components used, thus, minimizing the total cost of the scintillator. Typically, each experimental group does their own optimization of the scintillator composition per their needs. We hope that our work will assist all future experiments in this task.

Concentration and choice of dopants usually depends on the PMT sensitivity region. Contribution from either the fluor and shifter to the light yield are not linearly dependent on their amount in the solution. The optimization analysis of each component concentration on the output spectrum has been studied. Same optimization will apply to plastic-based scintillators as well.

\section{Optimization of fluor concentration}

Shown in Figure 1 is the setup used for PPO (2,5-diphenyloxazole) light yield measurements. It consists of two MELZ-FEU [2] PMT-115M, CAEN [3] DT5743 ADC and a PMT/sample holder. The ADC internally handles the double coincidence between the PMT signals. ${ }^{60} \mathrm{Co}$ was used with each sample. All measurements were conducted inside the black box.
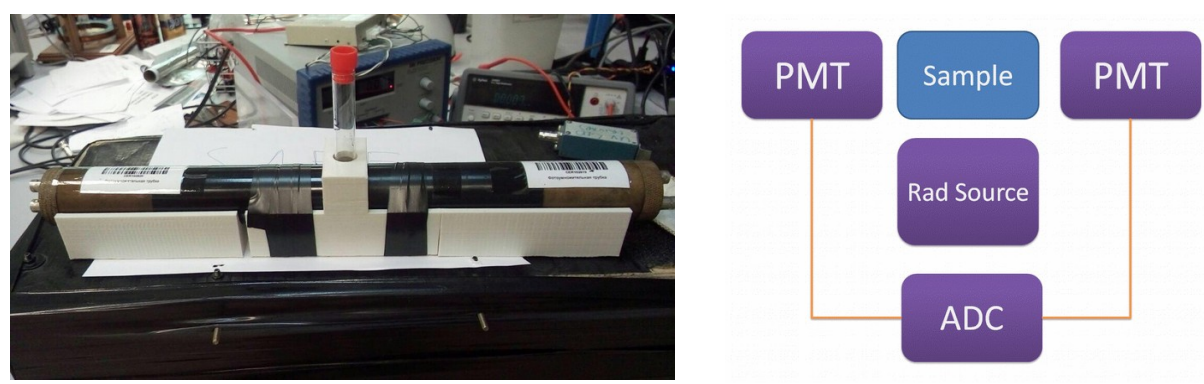

Figure 1: Light yeild measurmement setup (left) and setup schematics (right).

PC + PPO solution was used with the above setup. Histograms of the light intensity for different concentrations of PPO in the sample are shown in Figure 2.

The results of the analysis of light intensity dependence on PPO concentration are presented in Figure 3. They establish that there is no general-case optimal concentration for PC + PPO solution as the slope of the logarithmic function decreases monotonously. Sample with the PPO concentration $2 \mathrm{~g} / \mathrm{L}$ was chosen for future tests. 

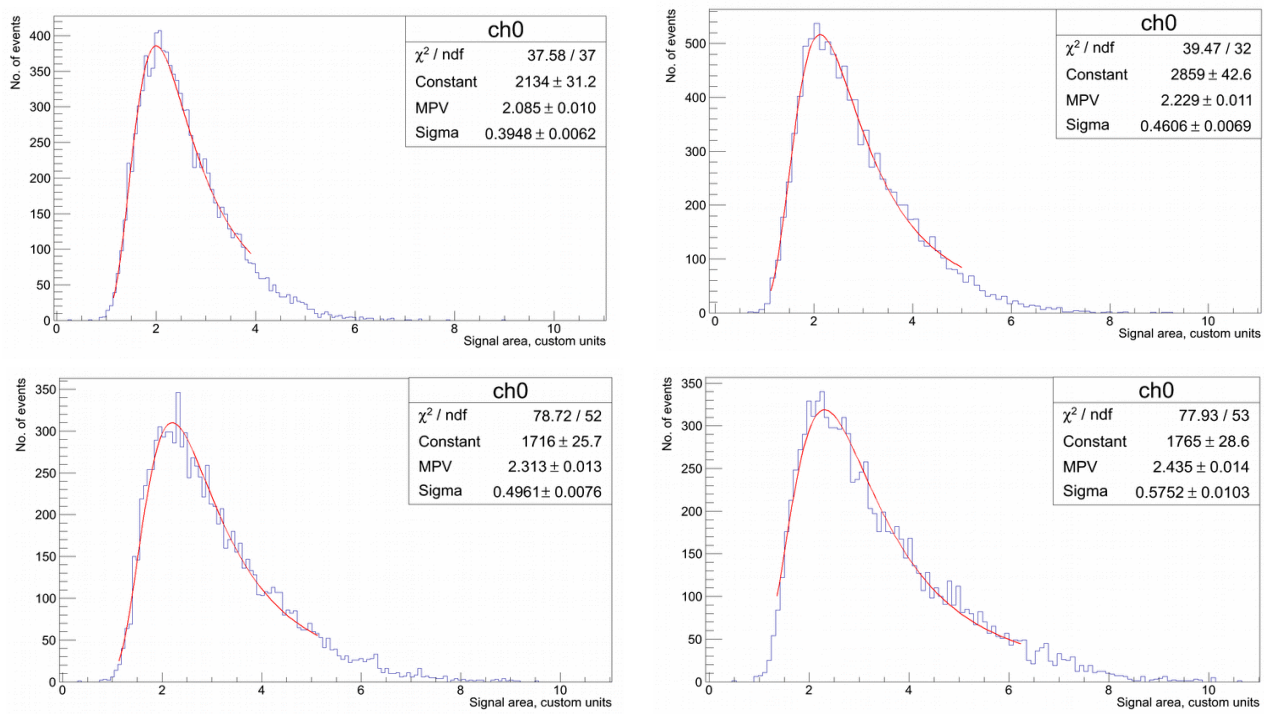

Figure 2: LS light yield with PPO concentrations $1 \mathrm{~g} / \mathrm{L}$ (top left), $1.5 \mathrm{~g} / \mathrm{L}$ (top right), $2 \mathrm{~g} / \mathrm{L}$ (bottom left), and $3 \mathrm{~g} / \mathrm{L}$ (bottom right)

The next step was to add shifter to observe how different concentrations will affect the light yield. Addition of MSB (1,4-Bis(2-mehylstyryl)benzene) to the sample caused the decrease in the total amount of light detected by PMTs. This unexpected result could be explained by the spectral sensitivity specifics of the MELZ-FEU PMT. It has very uniform sensitivity between $\sim 380 \mathrm{~nm}$ and $\sim 410 \mathrm{~nm}$, and in the first approximation the MSB is shifting the emitted by PPO light ( $\sim 380 \mathrm{~nm})$ within this range of wavelengths (to $410 \mathrm{~nm}$ ). This outcome has warranted detailed studies of the spectral distributions of LS light.

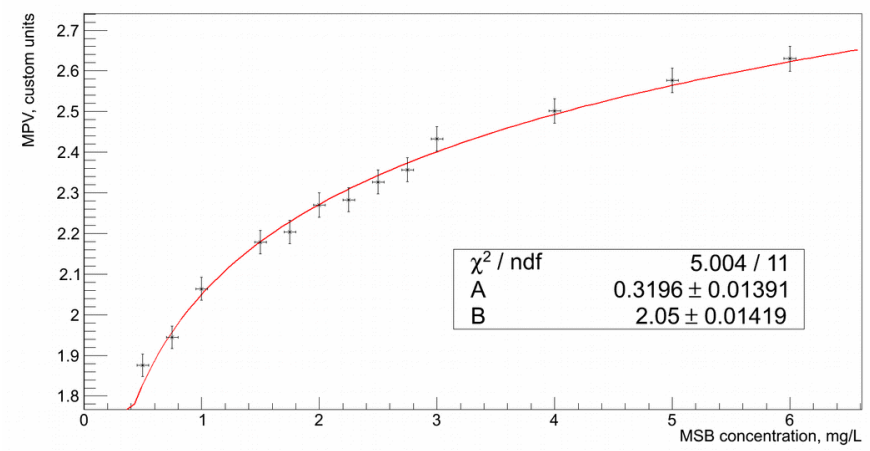

Figure 3: Light yield vs. PPO concentration

\section{Spectroscopy and MSB contribution}

Samples spectra for different concentrations of shifter in the solution were obtained. Measurements were done using Agilent [4] Cary Eclipse Fluorescence Spectrophotometer. In order to preserve the output spectra of the PPO as seen by the shifter and minimally affect the added shifter directly, the wavelength of the excitation light was chosen at $275 \mathrm{~nm}$ so that it will 
be absorbed by PPO only. The read-out range of the emitted light was chosen between 300-550 nm.

Changes in the shape of the spectra with different concentrations of MSB can be seen in the Figure 4. Contribution of the components of the solution is given by the parameters in the statistics box. The parameters of most interest are MSB_ex and MSB_em that represent the light absorbed and emitted by MSB. Ratio of these two parameters shows that only $40-50 \%$ of the light is re-emitted. Thus, integral light yield is reduced, supporting the explanation of the total light yield decrease seen in the section 2 .
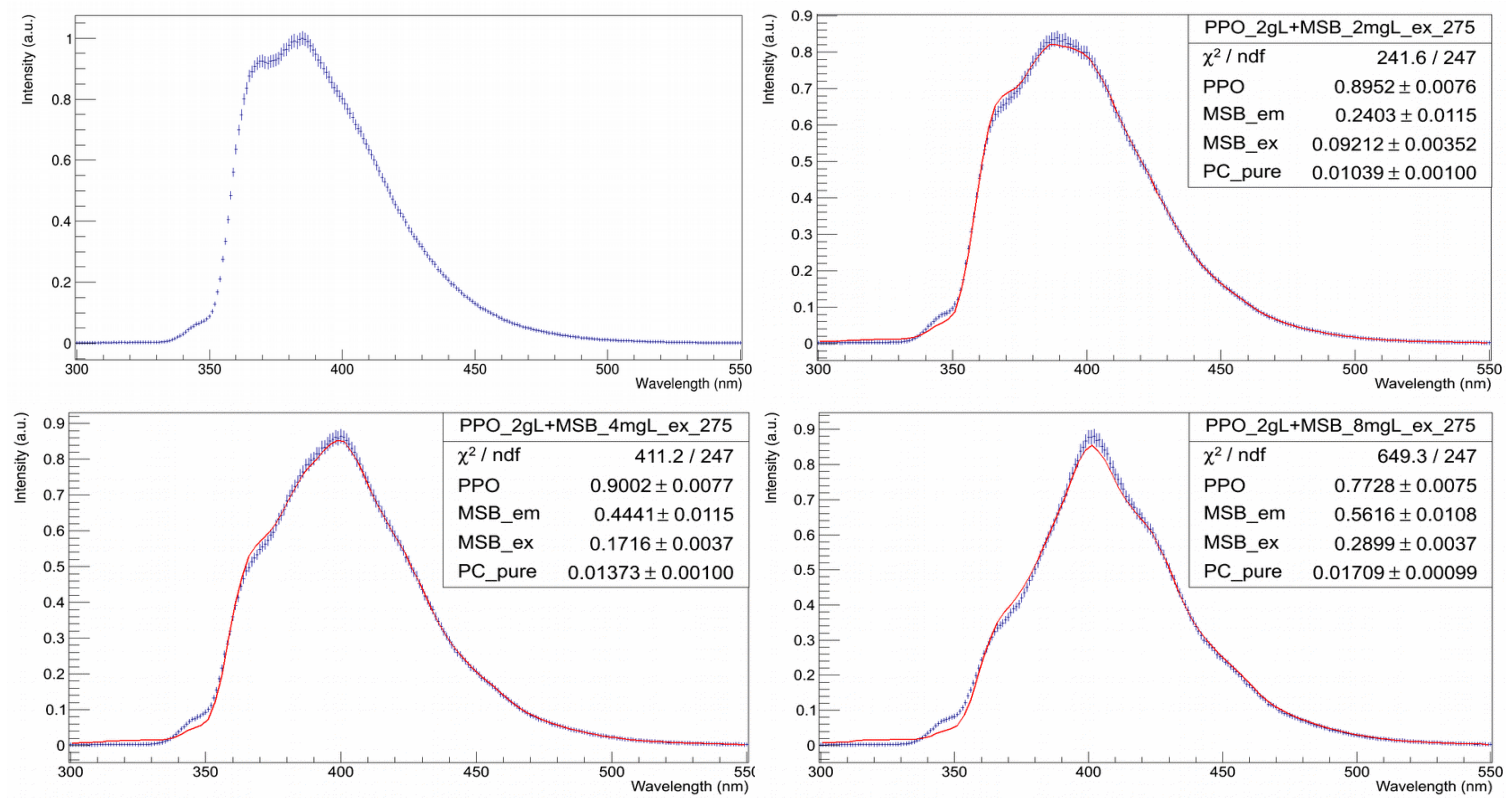

Figure 4: Change of output spectra. Pure PPO spectrum(top left), $2 \mathrm{mg} / \mathrm{L} \mathrm{MSB}$ (top right), $4 \mathrm{mg} / \mathrm{L} M S B$ (bottom left) and $8 \mathrm{mg} / \mathrm{L} \mathrm{MSB}$ (bottom right).

Data is fitted by the empirical equation to determine the contribution of each component. PPO, MSB_em, MSB_ex and PC_pure are the parameters for PPO emission light, MSB absorption, MSB emission, and PC/other background.

\section{Conclusion}

The work has been done on the optimization of LS composition. The results can also be applied to plastic scintillators as well. Concentration of each LS component can be chosen based on the needs from the fit parameters of the light yield. Experiment showed that the amount of output light is reduced when the shifter is added to base + fluor solution as shifter efficiency is $\sim 45 \%$. The choice of the shifter depends on the sensitivity of the detector used for the experiment. This study plans to continue the work using the novel water-based liquid scintillators [5]. 


\section{References}

[1] A Baitenov et al., Technical manual: a survey of scintillating medium for high-energy particle detection, arXiv:1601.00086, 2016/1/1

[2] MELZ-FEU, 4922-y pr-d, 4c5, Zelenograd, g. Moskva, Russia, 124482 (http://www.meltz-feu.ru)

[3] CAEN S.p.A. Via della Vetraia, 11, 55049 Viareggio Lucca, Italy (http://www.caen.it)

[4] Agilent INC., 5301 Stevens Creek Blvd, Santa Clara, CA 95051, United States (http://agilent.com)

[5] L. J. Bignell, D. Beznosko, M. V. Diwan, S. Hans, D. E. Jaffe, S. Kettell, R. Rosero, H. W. Themann, B. Viren, E. Worcester, M. Yeh, and C. Zhang. "Characterization and Modeling of a Water-based Liquid Scintillator", Journal of Instrumentation, Vol. 10, Pg 12009, IOP Publishing $(12 / 2015)$ 